\title{
Potential Demand Reduction from Buildings in a Simulated Utility
}

\author{
Brett Bass \\ Bredesen Center \\ The University of Tennessee \\ Knoxville, TN, US \\ bbass11@vols.utk.edu
}

\author{
Joshua New ${ }^{\dagger}$ \\ Building Technologies Research and Integration Center \\ Oak Ridge National Laboratory \\ Oak Ridge, TN, US \\ newjr@ornl.gov
}

\begin{abstract}
The availability of large-area sensing, scalable algorithms, and high-performance computing has enabled the possibility of urbanscale building energy modeling using new methods not limited to the scalability of manual building energy model creation or retrieval of county-by-county tax assessor's data. Automatic Building detection and Energy Model creation (AutoBEM) has created 178,368 building energy models for the Electric Power Board (EPB) of Chattanooga, TN, and compared simulation performance to 15-minute data from each building. These models leverage several data sources (e.g. imagery, GIS, utility), software tools to extract building properties (e.g. footprint, height, façade type, window-to-wall ratio, occupancy, building type), simulate at scale on two of the world's \#1 fastest supercomputers, and provide web-based visual analytics.

Grid-interactive efficient buildings offer the potential to reduce utility and rate-payer energy costs during each calendar month's hour of critical generation - when the least efficient, most costly, and often dirtiest generation assets must be brought online. In this paper, EnergyPlus is used to simulate over 150,000 buildings to assess the technical potential of utility-controlled smart thermostats. This is analyzed under a couple scenarios leveraging buildings as thermal batteries via pre-conditioning to coast through peak hours. Results are provided in box and whisker plots assessing the distribution of peak demand reduction at the utility-scale along with breakouts of energy and demand savings by building type and vintage.
\end{abstract}

\section{KEYWORDS}

Urban scale building energy modeling, Peak demand reduction, Smart thermostats

\footnotetext{
${ }^{\dagger}$ Corresponding author

Publication rights licensed to ACM. ACM acknowledges that this contribution was authored or co-authored by an employee, contractor or affiliate of the United States government. As such, the Government retains a nonexclusive, royalty-free right to publish or reproduce this article, or to allow others to do so, for Government purposes only. UrbSys '19, November 13-14, 2019, New York, NY, USA () 2019 Copyright held by the owner/author(s). Publication rights licensed to the Association for Computing Machinery.

ACM ISBN 978-1-4503-7014-1/19/11 ..\$15.00

https://doi.org/10.1145/3363459.3363526
}

\section{ACM Reference format:}

Brett Bass and Joshua New. 2019. Potential Demand Reduction from Buildings in a Simulated Utility. In 1st ACM International Workshop on Urban Building Energy Sensing, Controls, Big Data Analysis, and Visualization (UrbSys'19), November 13-14, 2019, New York, NY, USA. ACM, New York, NY, USA, 5 pages.

https://doi.org/10.1145/3363459.3363526

\section{Introduction}

High-performance computing and machine learning have led to new capabilities for processing large amounts of data. Among the possible capabilities is urban-scale building energy modeling. Obtaining building footprints from deep learning computer vision segmentation algorithms and building height from massive LiDAR datasets are two critical features of several that enable the creation of useful external details for building energy models.. The team has developed and applied such processing capabilities under the term Automatic Building detection and Energy Model creation (AutoBEM) [1][2]. Partnering with the Electric Power Board (EPB) of Chattanooga, TN, the corresponding building energy simulations can be compared to 15-minute electricity data from smart meters 178,368 real buildings. These serve to empirically validate and quantify subhourly, building-specific accuracy of each model. Digital twinning of a utility via processing of imagery (satellite and street-level) and other data sources for buildingspecific properties with custom software is beyond the scope of this study, but the interested reader is referred to [3][4].

By creating an error-informed digital twin of all buildings in the service area, any technology or policy can be assessed to determine building-specific and utility-scale information regarding the impact in terms of energy, demand, emissions, and financials. We apply Energy Conservation Measures (ECMs) as well as measures that impact demand and other elements related to building performance. ECMs applied include reducing building infiltration, improving HVAC efficiencies, reducing lighting power density, increasing insulation, and swapping out different HVAC and water heater system types. While these ECMs reduce the utilities energy use and thereby overall peak demand, they only indirectly impact the maximum monthly generation. Utilities are especially interested in reducing peak demand since it is costly, both to utilities and their 
customers, when they must utilize their least efficient and most costly generation assets.

Utility controlled smart thermostats are one such ECM that directly addresses demand reduction. Such a setup allows buildings to be used as thermal batteries. This is aided by pre-cooling or preheating the building prior to the peak hour and then coasts through the critical hour. Which buildings are pre-conditioned, at different times, and by different amounts is an ongoing area of interest.

For this analysis, EnergyPlus is used to simulate more than 150,000 buildings to evaluate the potential for grid-interactive buildings in the form of smart thermostats with multiple offsets. The results are visualized in box and whisker plots of percent demand reduction across the EPB service area as well as by building type and vintage. These demand reduction figures can be viewed as not only a cost saving measure to the utility and the ratepayer, but also as a reduction in emissions as the least efficient means of generation will be less utilized.

\section{Methodology}

The first step that had to be taken in building energy models for the EPB service area was collecting data that described the building's physical makeup. The two things necessary for this are building $2 \mathrm{D}$ footprint and building height. These two features provide a general shape to the building. These footprints were then associated with an electrical meter by finding the smallest Euclidean distance between each meter's GPS coordinates and the detected buildings. Building type and vintage were assessed by comparing the 15minute electricity use to the 97 prototype buildings and vintage combinations for this climate region (ASHRAE-169-2006-4A). Various other factors were calculated from these values and from there, building energy models could be created.

The models were created and simulated using two of the world's \#1 fastest supercomputers, Jaguar and Titan. The baseline simulation results were compared to the real electricity data from EPB for quality assurance. By using a crude approach of assigning building type as small, medium, or large office and comparing to 15-minute energy data, we achieved CV(RMSE) $15=205.9 \%$ and $\mathrm{NMBE}_{15}=10.1 \%$ [5]. ECMs, once defined properly, can be used to modify all buildings, simulated on HPC resources, output transferred to analysis node, analyzed, and summarized with interactive, online analytics within 6.5 hours. This paper focuses on the demand-reducing measure for smart thermostats under scenarios where each building model is simulated at a four and eight degree $(\mathrm{F})$ offset. The utility requested these setback values with a target of eight degrees or higher for potentially unoccupied or abandoned buildings.

We use actual meteorological year (AMY) weather data for Chattanooga, TN, that corresponds to the time period for which 15minute data has been provided. Based on that, we look up the
HVAC mode and temperature at the peak hour to determine whether heating or cooling are needed for proper pre-conditioning. We note that while this clairvoyance may be unrealistic in practice, most buildings (even during shoulder months) are typically in a clear heating or cooling mode both before and at the peak hour (which often is in the cold morning on cool months and the hot evenings during warm months). For the four-degree offset, there is a two hour pre-cool or pre-heat period where the thermostat setpoint is the average of the existing cooling and heating setpoint temperature with an added 0.5 degree (C) deadband to avoid hysteresis. Then for the peak demand hour and the following four hours after the peak, the cooling and heating setpoints increased and decreased by four degrees respectively.

For the eight-degree offset, the approach is the same as the 4-degree offset with only minor differences. The pre-cool or pre-heating period increase to four hours prior to the peak demand hour. In addition, the cooling and heating setpoints increased and decreased by eight degrees respectively for the peak demand hour and the following four hours after the peak.

\section{Results}

\subsection{All Buildings}

The results are reported in by percent demand reduction and are shown in box and whisker plots. The results are in three formats for the four- and eight-degree offsets; All buildings, grouped by building type, and grouped by vintage. Figures 1 and 2 contain all buildings in the service area.

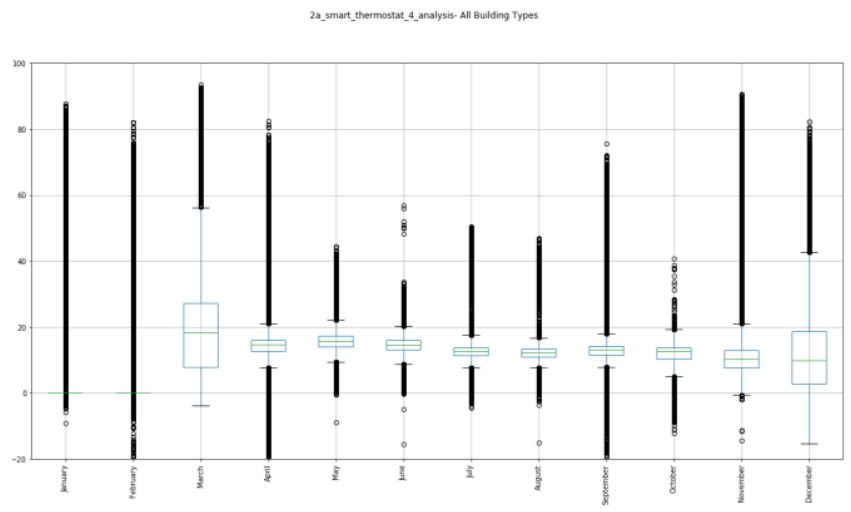

Figure 1. Average peak demand reduction across all 178,368 buildings is $13 \%$ under a $4^{\circ} \mathrm{F}$ pre-conditioning scenario.

The overall average peak demand reduction over the course of the year is $13.19 \%$. The summer transitional months (March, April, May) tend to have the highest percentage demand reduction with March having the highest average percentage demand reduction of $22.28 \%$ for the 4-degree scenario. It should be noted that these shoulder months have less demand, so while the relative percentage may be higher, the absolute $\mathrm{kW}$ is typically lower compared to other months. The average percent demand reduction stays 
relatively constant throughout the summer before dipping to a local minimum in October. The average percent demand reduction then increases in the winter transitional month (November) before decreasing to the global minimum of the winter in January and February.

March and November tend to have the highest variance with March having a variance of more than 3.5 times the average variance and November having a variance of more than 2.5 times the average.

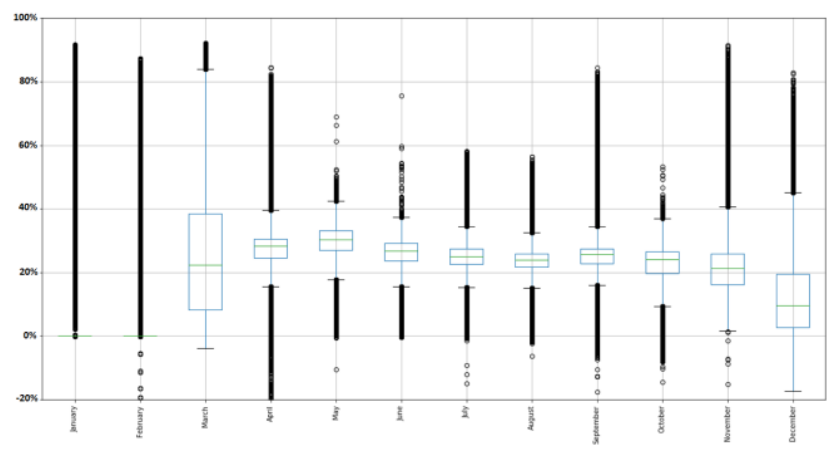

Figure2. Average peak demand reduction across all 178,368 buildings is $22 \%$ under an $8^{\circ} \mathrm{F}$ pre-conditioning scenario.

2a_smart_thermostat_8_analysis
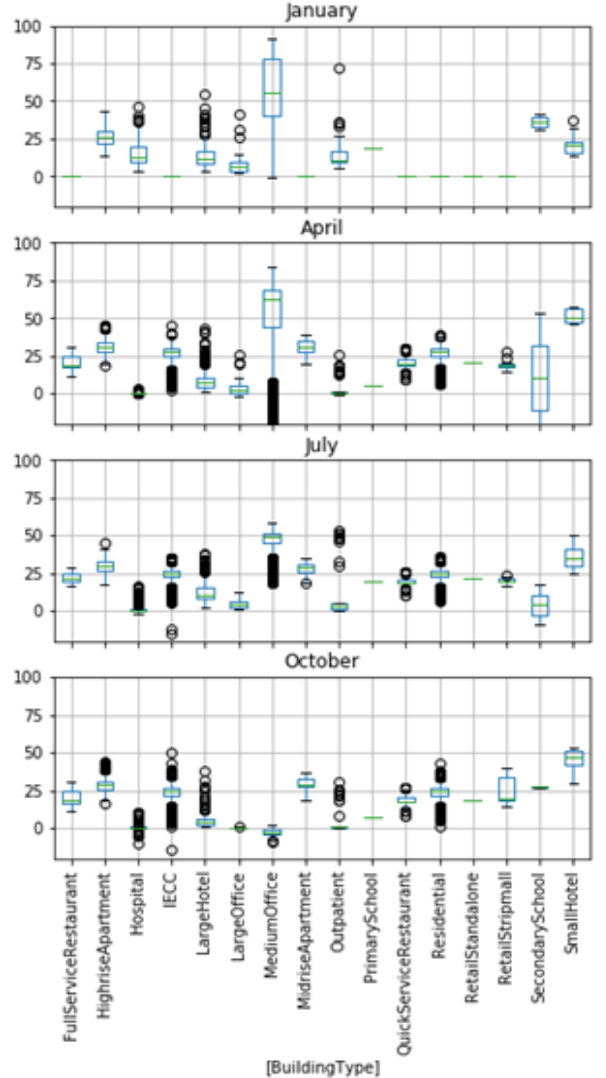

The eight-degree smart thermostat offset has a similar shape to the four-degree offset. As should be expected, eight-degree demand savings is higher than four-degree demand savings, but is not twice the four-degree amount. The overall average peak demand reduction over the year is $21.70 \%$ (60\% more than 4-degree). Similarly, the summer transitional months have the most percent peak demand reduction with April being the highest at $29.35 \%$. Again the demand stays somewhat constant throughout the summer with the local minimum occurring in October before rising in the winter transitional month of November. The percent demand reduction then falls to the yearly minimum in January and February.

March and November still have the high variance among buildings with March varying by more than 3.05 and 1.44 times the average variance respectively. Although, for the eight-degree case, January and February also has a large variance at 2.01 and 1.68 times the average variance respectively.

\subsection{By Building Type}

As the structure of the percent demand reduction plots for four and eight degrees are similar except on a different scale, only the eightdegree plots are shown when grouped by building type and vintage so the striation can be better seen. The four-degree figures are
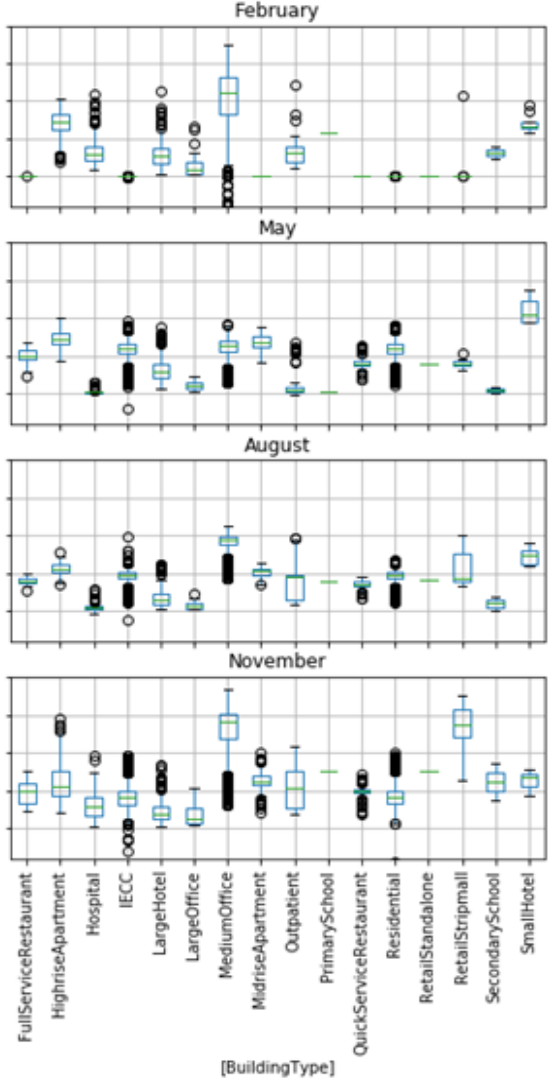
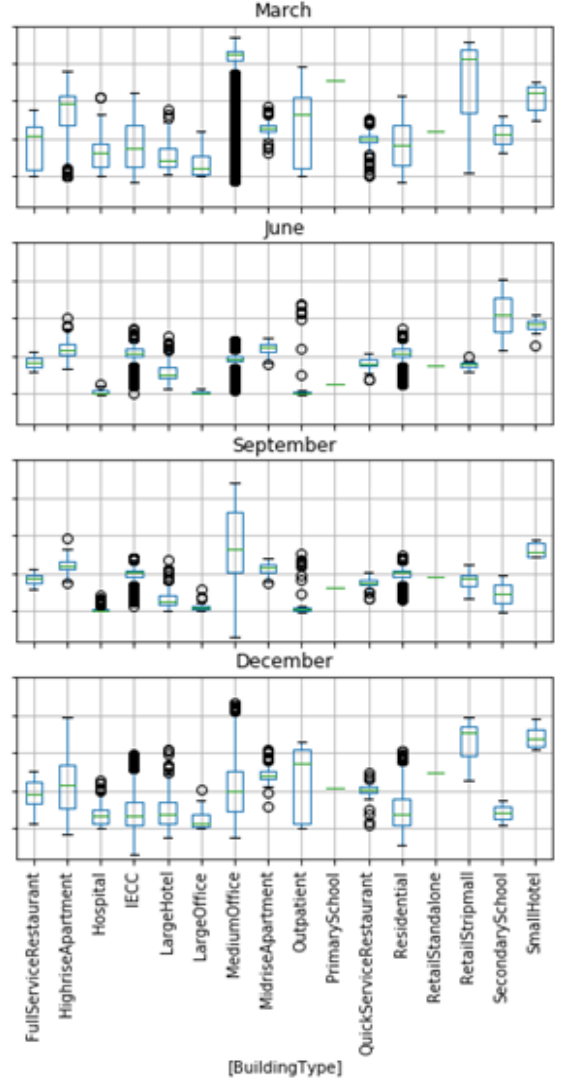

Figure 3. Each building type is shown with the quartile ranges and outliers for each calendar month. This could enable empirical validation for what are indicated to be high-value building types (e.g. common medium offices and retail strip malls) and times. 

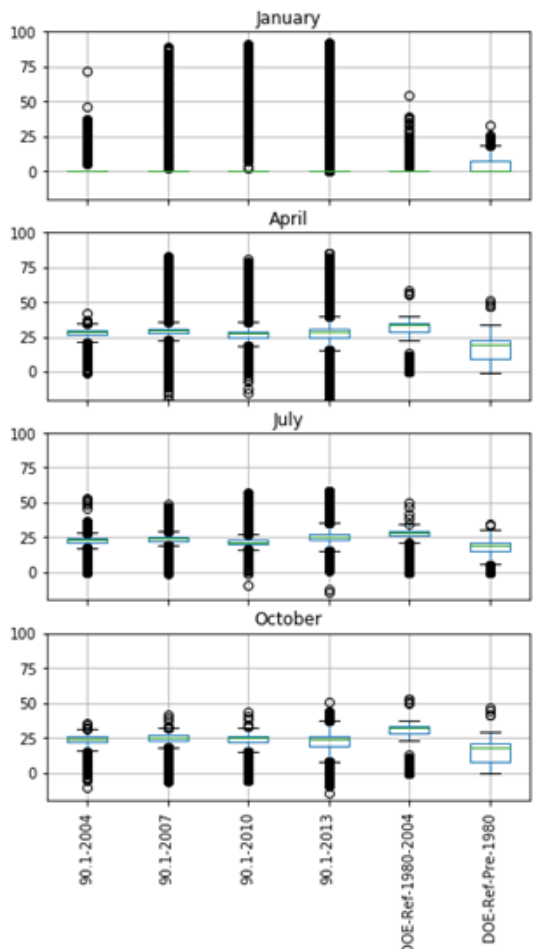

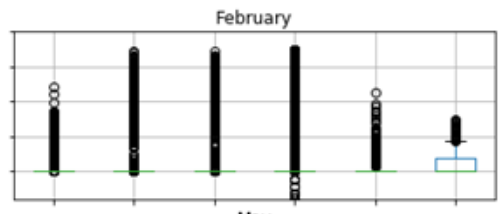

May

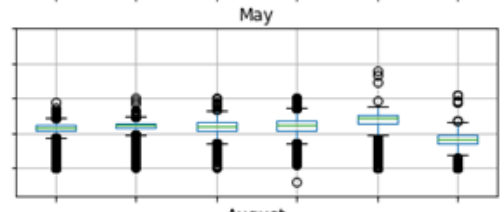

August

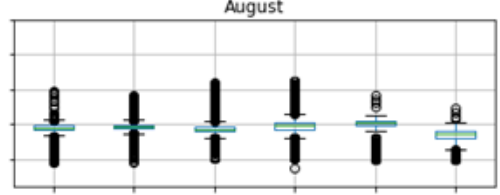

November

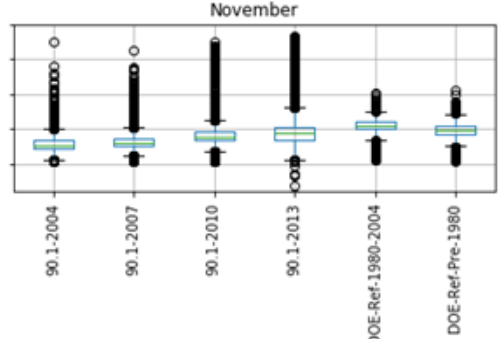

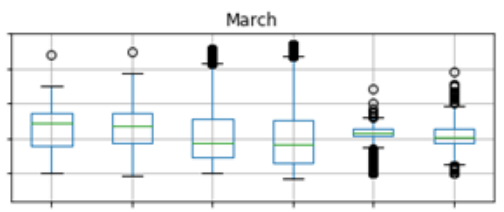

june

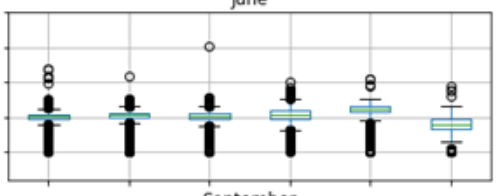

September

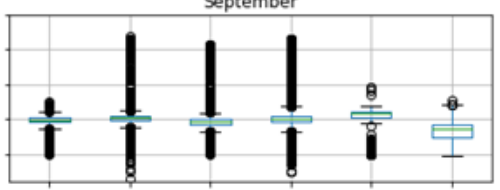

December

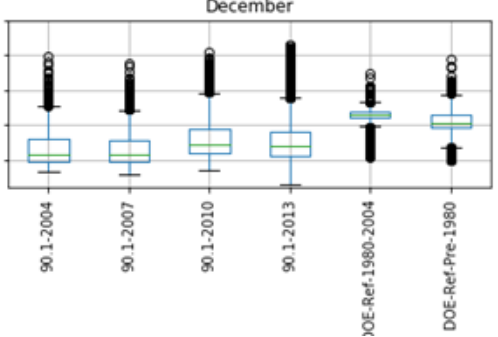

Figure 4: Relative demand reduction potential for each vintage (across all building types) slightly increases with newer vintages.

located in the appendix, Figure A1. Figure 3 contains the percent peak demand reduction grouped by building type.

The trend for percent demand reduction for each of the building types is similar to the overall trend for all buildings. There are a couple building types that have somewhat different patterns. As expected, medium office it's highest reduction potential in March but interestingly, this building type also has very high demand reduction potential in January and February compared to the summer months which is opposite the total building trend. It is of note that many buildings including residential (IECC) have close to zero demand reduction with almost no variance. This could be due to the use of gas for heating while it was cold in these months in this particular year, but is certainly an artifact of the building energy models. Residential should be noted as more than $80 \%$ of building types in the EPB service area are residential. It appears that the residential buildings follow the overall building trend closely.

Slicing the data by building type enables identification of lucrative buildings and times for maximum peak reduction. The building type with the most potential for reduction is the medium office which has consistently higher percent demand reduction than the other building types. The average percent demand savings for a medium office in March are well over $75 \%$. After medium office, retail strip malls have the highest demand reduction potential.

\subsection{By Vintage}

As in Section 3.2, only the eight-degree plots are shown for the vintage comparison. The four-degree figures are located in the appendix, Figure A2. Figure 4 contains percent peak demand reduction grouped by vintage.

The trend with each vintage is similar to the overall building trend with March and the summer transition months being the highest in general and the winter months (January and February) being the lowest.

While most vintages follow the overall trend, it is interesting to note that vintages before 2004 have higher percent peak demand reduction in December. The authors conjecture that this may be due to the higher infiltration rates in older buildings with a setback temperature that reduces the exfiltration of heating during cold months. It is encouraging to note that newer vintages have a higher relative demand savings potential than older buildings. Such buildings are also likely to have other sensors or equipment that could respond to grid signals.

\section{Conclusion}

A digital twin of buildings in a utility's service area was simulated using EnergyPlus, validated with real 15-minute whole-building electricity data, and used to assess the potential of grid-interactive smart thermostats for managing monthly peak demand. 
The BEM-based assessment indicates smart thermostats with a 4degree offset resulted in an average of $13.19 \%$ reduction in peak demand over the year while a 4-degree offset resulted in an average of $21.70 \%$ reduction in peak demand over the year. The summer transition months offered the highest potential for demand reduction while the winter months offered the lowest.

The building type with the highest potential for grid-interactive smart thermostat demand reduction was clearly medium office while other buildings offered similar reduction values over the course of the year. Both commercial and residential building types had nearly zero demand reduction in the months of January and February. The trend amongst the different vintages was similar to the overall trend with the exception of older buildings (pre 2004) having higher potential to shave peak demand in the month of December.

There is much ongoing and future work involving scalable and cost-permissive extraction of building-specific properties being investigated by the authors. It is known that building energy use can vary by $3 x$ per year or $5 x$ per month between an energy saver and an energy waster in a similar building. Occupant behavior is a significant remaining source of uncertainty in building models, is difficult to address in light of privacy concerns, and is an area of active exploration by several funding agencies. Enhanced assignment of occupancy, further comparison to measured data, testing and improvement of different data layers/sources to quantify reduction of modeling errors, and empirical validation of predicted energy savings are all activities being actively explored by the authors.

\section{ACKNOWLEDGMENTS}

This work was funded by field work proposal CEBT105 under DOE Building Technology Activity Number BT0201000. This research used resources of the Oak Ridge Leadership Computing Facility at ORNL, which is supported by the Office of Science of the DOE under Contract No. DE-AC05-00OR22725.

ORNL is managed by UT-Battelle, LLC, for DOE under contract DE-AC05-00OR22725. This manuscript has been authored by UTBattelle, LLC, under Contract Number DEAC05-00OR22725 with DOE. The United States Government retains and the publisher, by accepting the article for publication, acknowledges that the United States Government retains a non-exclusive, paid-up, irrevocable, world-wide license to publish or reproduce the published form of this manuscript, or allow others to do so, for United States Government purposes.

\section{REFERENCES}

[1] New, Joshua R., Adams, Mark, Im, Piljae, Yang, Hsiuhan, Hambrick, Joshua, Copeland, William, Bruce, Lilian, Ingraham, James A. (2018). "Automatic Building Energy Model Creation (AutoBEM) for Urban-Scale Energy Modeling and Assessment of Value Propositions for Electric Utilities." In Proceedings of the International Conference on Energy Engineering and Smart Grids (ESG), Fitzwilliam College, University of Cambridge, Cambridge city, United Kingdom, June 25-26, 2018. [PDF] [PPT]

[2] New, Joshua R, Bhandari, Mahabir, Shrestha, Som, and Allen, Melissa. (2018). "Creating a Virtual Utility District: Assessing Quality and Building Energy Impacts of Microclimate Simulations." In Proceedings of the International Conference on Sustainable Energy and Environmental Sensing (SEES), Cambridge, UK, June 18-19, 2018. [PDF] [PPT]
[3] New, Joshua, Copeland, William, and Ingraham, James (2018). "Poster: Virtual Electric Power Board of Chattanooga, TN (EPB)." ORNL BTO Peer Review, April 15, 2019. [Poster]

[4] Ingraham, James A. and New, Joshua R. (2018). "Virtual EPB." Presented to Building Technologies Office following the BTO Peer Review, 87 slides. Arlington, VA, May 3, 2018. [PPT]

[5] Garrison, Eric, New, Joshua R., and Adams, Mark (2019). "Accuracy of a Crude Approach to Urban Multi-Scale Building Energy Models Compared to 15-min Electricity Use." Best PhD Student Paper award. In Proceedings of the ASHRAE Winter Conference, Atlanta, GA, Jan. 12-16, 2019. [PDF] [PPT] 